\title{
A repercussão do diagnóstico de HIV positivo em homens: um estudo qualitativo
}

\author{
The repercussion of the HIV-positive diagnosis in men: a qualitative study
}

Las repercusiones del diagnóstico de VIH en los hombres: un estudio cualitativo

Daniele Cabral Dias ${ }^{1 *}$, Olívia Paulino Pinto ${ }^{1}$, Aline Rodrigues Feitoza1, Danielle Teixeira Queiroz¹, Francisco Gabriel de Andrade Mota ${ }^{1}$, João Victor Farias Mota ${ }^{1}$, José Reginaldo Pinto ${ }^{1}$, Régila Maria Farias Pinto ${ }^{1}$, Valéria Freire Gonçalves ${ }^{1}$, Geysa Maria Nogueira Farias².

\section{RESUMO}

Objetivo: Analisar o significado do diagnóstico de HIV positivo para homens e as suas possíveis repercussões emocionais. Métodos: Trata-se de um estudo qualitativo. A coleta foi realizada mediante entrevista semiestruturada, gravada após consentimento com 20 homens, no período de janeiro a março de 2019 em um serviço de atenção secundária situado no estado do Ceará. A análise se dividiu em duas fases, uma temática e outra interpretativa, foi usado referencial teórico do Interacionismo Simbólico. Resultados: A partir da análise dos depoimentos foram identificadas as seguintes categorias: rejeição e a omissão no conviver com a infecção pelo HIV, que se divide em duas perspectivas em relação ao processo de conviver com o HIV e o significado de ter HIV antes e após o conhecimento aprofundado da doença. Conclusão: Ainda existem dificuldades relacionadas ao nível de conhecimento da doença, o estigma e a omissão da HIV. Porém, é possível, assim como foi relatado a mudança do significado.

Palavras-chave: Interacionismo simbólico, Homens, HIV.

\begin{abstract}
Objective: To analyze the meaning of the HIV-positive diagnosis for men and its possible emotional repercussions. Methods: This is a qualitative study. The collection was conducted through semi-structured interviews, recorded after consent with 20 men, in the period from January to March 2019 in a secondary care service located in the state of Ceará. The analysis was divided into two phases, one thematic and one interpretative, the theoretical referential of Symbolic Interactionism was used. Results: From the analysis of the statements the following categories were identified: rejection and the omission in living with HIV infection, which is divided into two perspectives in relation to the process of living with HIV and the meaning of having HIV before and after the in-depth knowledge of the disease. Conclusion: There are still difficulties related to the level of knowledge of the disease, the stigma and the omission of HIV. However, it is possible, as reported, to change the meaning.
\end{abstract}

Keywords: Symbolic interactionism, Men, HIV.

\section{RESUMEN}

Objetivo: Analizar el significado del diagnóstico de VIH positivo para los hombres y sus posibles repercusiones emocionales. Métodos: Se trata de un estudio cualitativo. La recolección se realizó a través de entrevistas semiestructuradas, grabadas previo consentimiento con 20 hombres, en el período de enero a marzo de 2019 en un servicio de atención secundaria ubicado en el estado de Ceará. El análisis se dividió en dos fases, una temática y otra interpretativa, se utilizó el referencial teórico del Interaccionismo Simbólico. Resultados: A partir del análisis de las declaraciones se identificaron las siguientes categorías: el rechazo y

1 Universidade de Fortaleza (UNIFOR), Fortaleza - CE. *E-mail: dteixeiraqueiroz@yahoo.com.br

2 Núcleo de Atenção Médico Integrado (NAMI), Fortaleza - CE. 
la omisión en la vivencia de la infección por el VIH, que se divide en dos perspectivas en relación con el proceso de vivir con el VIH y el significado de tener el VIH antes y después del conocimiento profundo de la enfermedad. Conclusión: Siguen existiendo dificultades relacionadas con el nivel de conocimiento de la enfermedad, el estigma y la omisión del VIH. Por lo tanto, es posible que, al igual que el cambio de significado, sea posible.

Palabras clave: Interaccionismo simbólico, Hombres, HIV.

\section{INTRODUÇÃO}

A AIDS é uma condição causada pelo retrovírus HIV que pode contribuir significativamente para sequelas que vão além daquelas físicas e biológicas em quem a vivência, uma vez que, afeta a visão corporal que os indivíduos têm de si e do mundo, modificando as demais esferas da vida (SOARES MN, et al., 2019). E isso vem sendo cada vez mais observado diante do impacto que o diagnóstico do HIV traz na vida dos usuários, mexendo com sua perspectiva de futuro e nas relações afetivas e sociais (CATUNDA C, et al.,).

Nessa mesma linha de pensamento vale a pena destacar a trajetória invisível de muitos, que vivem com o HIV na quietude, sinalizado pela percepção de autoexclusão do convívio em sociedade, pelo estigma e a discriminação, e por diversos prejuízos de cunho emocional, tais como, crises de ansiedade, sintomas depressivos, desalento, isolamento individual e sentimentos autodestrutivos com grandes implicações psicológicas, econômicas e sociais e, em casos mais limiares com disposição e ideia suicida (CAMPOS ERA e NERY JSNN, 2015).

De acordo com o relatório do (Programa Conjunto das Nações Unidas sobre HIV/AIDS) em 2017, no mundo há aproximadamente 36,9 milhões de pessoas que vivem com HIV. Sendo que, houve um declínio de novas infecções pelo HIV de $47 \%$ desde do auge em 1996. Em 2017, foram identificados 1,8 milhão de novos casos de HIV em comparação com 3,4 milhões em 1996. A partir de 2010, as novas infecções por HIV em adultos baixaram cerca de $16 \%$, de 1,9 milhão para 1,6 milhão em 2017. No Brasil foram detectados 194.217 casos de infecção pelo HIV, na região Nordeste foram 14.275 (7,4\%). Com relação à raça/cor da pele autodeclarada, foram registrados $47,6 \%$ são brancos e $51,5 \%$ entre pretos e pardos. No gênero masculino foram notificados $49,6 \%$ casos em brancos e $49,4 \%$ entre pretos e pardos; entre as mulheres, $43,2 \%$ dos casos são entre brancas e 55,9\% entre pretas e pardas (BRASIL, 2017).

No estado do Ceará, no período de janeiro de 2011 a dezembro de 2020 foram diagnosticados 12.745 novos casos de HIV e 10.609 de Aids (CEARÁ, 2021). Desde 2015, vem sendo observado uma queda da taxa de detecção de Aids que passou de 12,8/100 000 habitantes em 2015 para 7,9/100.000 habitantes no ano de 2020, revelando uma redução de 38,2\% (CEARÁ, 2021). Esta redução da taxa de detecção tem sido evidente desde a recomendação do "tratamento para todos", implantada em dezembro de 2013 (PCDT, 2013). Entretanto, foi observado um acréscimo no número de casos novos da infecção pelo HIV até o ano de 2019, com decréscimo acentuado em 2020, provavelmente resultante da pandemia de COVID 19 que limitou os diagnósticos nos serviços de saúde (CEARÁ, 2021).

No período analisado, foram registrados no Sistema de Informação de Agravos de Notificação (SINAN), um total de 9.628(75,4\%) casos de HIV em homens e $3.142(24,6 \%)$ casos de HIV em mulheres (CEARÁ, 2021). A razão de sexo (razão $M / F$ ) apresentou pouca oscilação no período, sendo registrado 32 casos de HIV em homens para cada dez casos em mulheres no ano de 2020 (CEARÁ, 2021).

Nesse contexto, avaliando a vulnerabilidade da infecção pelo vírus do HIV no homem, nota-se que esse acometimento se deve à falta de conhecimento da prevenção da doença e no seu sentimento de invulnerabilidade, na qual, o torna mais exposto ao vírus do HIV. Dessa forma, faz-se necessário salientar a importância de uma atenção efetiva a respeito do tema e é importante o planejamento dos profissionais de saúde nas práticas educativas destes indivíduos (COSTA MAR, et al., 2019).

A Política Nacional de Atenção Integral à Saúde do Homem (PNAISH) foi instituída em 2009, mas ao se passar dez anos, ainda são visíveis as dificuldades na captação dessa população aos serviços de saúde 
(BRASIL, 2009). As ações de educação em saúde podem ser potencializadas pelo uso de metodologias participativas que incorporem elementos ligados às crenças, à moral, aos preconceitos, à afetividade e à história de vida dos sujeitos envolvidos nas ações de educação em saúde (GUIMARÃES DA, et al., 2017).

Os homens entendem o HIV como uma "sentença de morte" e consideram as pessoas com diagnóstico positivo como mortas, mostrando não apenas medo, mas também um sentimento de inutilidade frente à doença (DICARLO AL, et al., 2014). Diante da problemática apresentada surgiu o seguinte questionamento: Qual o significado do diagnóstico positivo de HIV para homens infectados?

O interesse pelo tema surgiu da inquietação das autoras acerca da incipiência de trabalhos científicos sobre o significado da vivência do HIV para o homem, além disso, estas se perguntaram como a figura masculina enfrenta o diagnóstico, visto o modelo hegemônico existente. Nessa perspectiva, esse estudo justifica-se pela alta incidência de HIV em homens, pela escassez de trabalhos com essa temática e por fim, pela necessidade da Enfermagem se apropriar desse conhecimento. E assim a pesquisa objetiva analisar 0 significado do diagnóstico de HIV para homens com sorologia positiva.

\section{MÉTODOS}

O cenário da pesquisa foi o serviço de atenção secundária que atende pessoas que vivem com HIV no Estado do Ceará. Trata-se de uma instituição de assistência e tratamento para pessoas vivendo com HIV/ AIDS em todo o estado do Ceará. As entrevistas aconteceram no período de janeiro a março de 2019, com 20 homens que vivem com HIV. Cada participante foi codificado com a letra "H" seguida de números de 1 a 20.

Foram elegíveis os indivíduos que apresentavam diagnóstico positivo de HIV a mais de 6 meses; idade maior que 18 anos e estar em acompanhamento na instituição por pelo menos um ano. Os critérios de exclusão foram possuir algum tipo de deficiência mental, que os incapacitou de responder a pesquisa. Inicialmente não houve definição prévia da quantidade de participantes do estudo, finalizando a coleta a partir da saturação teórica dos depoimentos, ou seja, quando nenhum elemento novo emergir do material empírico.

As entrevistas foram realizadas individualmente numa sala reservada com o intuito de preservar as individualidades e singularidades dos participantes no ambulatório de infectologia. Essas foram gravadas e duraram em média 32 minutos e sendo feitas com o apoio de um roteiro de apoio semiestruturado contendo as seguintes perguntas norteadoras: "O que significou para você o diagnóstico da infecção pelo HIV?"; "Após o diagnóstico positivo de HIV, quais foram as mudanças ocorridas na sua vida social?"; "Após o diagnóstico positivo de HIV, quais foram as mudanças ocorridas na sua vida familiar?"; "O que você sabia sobre o HIV antes de ser diagnosticado e durante o tratamento?" Você considera que sua visão sobre o HIV mudou após o diagnóstico? Se sim, alguém teve influência nessa mudança".

Para a captação e convite dos participantes, as pesquisadoras abordaram- os na sala de espera para a sua consulta de acompanhamento mensal com a enfermeira e o médico infectologista e após o aceite foram direcionados para o local reservado. Neste, havia a explicação dos objetivos da pesquisa e consequentemente leitura e assinatura do Termo de Consentimento Livre e Esclarecido (TCLE), assegurando-se o sigilo e a confidencialidade dos depoimentos. A pesquisa foi aprovada no Comitê de Ética em Pesquisa sob o parecer de número 3.517.843.

Chegou-se às temáticas, através da análise dos temas que trabalha com núcleos de sentido, em que o pesquisador deve identificar se a presença ou frequência de certo tema significa alguma coisa para o objeto analítico, este tipo de análise possui três etapas: a pré-análise; exploração do material e tratamento dos resultados obtidos e interpretação.

\section{RESULTADOS E DISCUSSÃO}

\section{Rejeição e a omissão no conviver com a infecção pelo HIV}

A primeira temática traz duas perspectivas em relação ao processo de conviver com o HIV: a primeira perspectiva é o viver com o HIV em sociedade, visto que os participantes expressavam um sentimento de 
medo, uma preocupação em relação ao seu diagnóstico se tornar público e ficar sujeito a estigmas. Mostrando que, a infecção interferiu diretamente no seu convívio social, interrompendo assim, sua qualidade de vida. Tal fato é evidenciado nas falas a seguir:

"Vivo normal, mas tem a questão do preconceito das pessoas que não entendem o que é o HIV" (H2).

"Eu, fiquei assim surpreso. Mas, assim sem querer acreditar, né. Mas tinha que acreditar, até porque eu não comentei com ninguém. Fica só entre mim" (H4).

"Se eu digo eu posso correr o risco de sofrer a rejeição e eu tenho uma certa dificuldade com a rejeição, a gente sempre tem, né" (H9).

Diante disso, estudos sobre os significados e interpretações para a interação social, ressaltam que a compreensão de cada pessoa sobre a situação está intimamente ligada com a forma pela qual essa irá reagir durante todo o a história natural da doença sendo essa relação indispensável para a um bom processo de manutenção de toda a vida. (LENZI L, et al., 2018).

No entanto, ao analisar os depoimentos percebe-se a partir deles, que o fato de ter HIV coloca-os numa situação diferenciada, levando-os ao sofrimento, resultante da rejeição e discriminação que o significado construído impõe, interferindo na sua inserção na sociedade. A sociedade produz um sentimento de intolerância a respeito das pessoas soropositivas, que impede seu desenvolvimento como ser humano devido ao desconhecimento sobre o HIV e acabam se posicionando de maneira inadequada manifestando opiniões negativas, oprimindo aqueles que são acometidos pela doença (JESUS GJ, et al., 2017).

Nessa condição, a prática de tais ações faz com que muitos indivíduos com HIV encaram a doença sozinhos sem o apoio dos amigos, família ou qualquer um dos ciclos que lhe circundam acaba prejudicando sua interação com a sociedade o que os leva a ocultar sua condição sorológica pelo medo de sofrer preconceito ou discriminação, fomentada pelo estigma (FREITAS MIDF, et al., 2017).

Apesar do conhecimento sobre o HIV estar presente em diversos formatos: internet, televisão, livros, persiste em nossa sociedade sentimentos conflituosos e preconceituosos em relação a essa doença. Diante das falas dos participantes, foi observado a omissão da doença para a família e a percepção de desconforto dos familiares diante da descoberta da doença.

Na perspectiva seguinte, a família é a entidade de maior receio em relação a descoberta do diagnóstico, em virtude dos vínculos e do preconceito desvelado.

"Minha família não sabe, mas desconfia" (H2).

"Preocupação com a minha família, como que eles iam responder a isso, que eu tenho até hoje, porque eles não sabem ainda" (H13).

"Não, na realidade ninguém sabe, mesmo porque você sabe que hoje em dia o preconceito ele ainda é muito forte, entendeu? Mesmo eu... assim, as pessoas da área da saúde e as pessoas que tem sabe que só é transmitido no contato sexual ou com sangue, você não pega no beijo, você não pega no abraço, no aperto de mão, mas até você explicar isso para a sociedade é complicado, né?" (H14).

"Não, praticamente só Deus mesmo, só Deus porque a família, eu vou ser bem sincero, a família tenta aceitar, mas sempre fica aquele negócio meio esquisito, não é? Não consegue totalmente...tenta disfarçar né? Eu não entro em atrito porque não vai valer a pena, eu vou me estressar, essas coisas todas. Mas eu vejo que tem um pouco de receio deles e aí gente leva, né?" (H19).

Inicialmente não, porque só quem soube foram duas amigas, então eu teoricamente não vi mudança porque os demais não... Não sabem. Ninguém sabe. (H15) 
De acordo com o interacionismo simbólico o ser humano age em relação a algo a partir da percepção do indivíduo sobre a situação, ou seja, a omissão da doença diante dos familiares, apenas reforça a ideia negativa que foi construída pelas pessoas sobre o HIV (LENZI L, et al., 2018).

Assim, a família, vista como principal rede de apoio, tornou-se excluída de todo o processo, do diagnóstico ao tratamento. As pessoas que vivem com HIV tendem a revelar mais doenças para a família extensa do que a nuclear, consequentemente, eliminando uma importante fonte de apoio emocional e formação de resiliência. A família e os amigos configuram-se como fatores essenciais de proteção contra 0 isolamento social e possíveis transtornos mentais (FREITAS MIDF, et al., 2017).

Ainda nesse escopo, a rede familiar possui participação decisiva na adesão dos antirretrovirais, nas mudanças de rotina e hábitos e por fim na adaptação das medicações que possuem os mais diversos efeitos colaterais. A omissão da verdade elimina o potencial familiar de reconstruir e mudar o significado do HIV.

\section{O significado de ter HIV antes e após o conhecimento aprofundado da doença}

No momento em que foram interrogados sobre seus conhecimentos prévios antes de serem acometidos pelo HIV, alguns dos homens entrevistados relataram que possuíam conhecimento superficial sobre a doença. Que se representa claramente nas falas a seguir:

"Se fala muito em HIV, AIDS e morte. Não se fala nesses detalhes né" (H9).

"Eu sabia antes que é uma coisa complicada, a pessoa tinha isso e já era AIDS, aí morria aquele negócio todo, né? Morria magro, daquele jeito, né? Que as pessoas imaginam" ( $\mathrm{H} 18)$.

"Eu sabia que era pra sempre, eu sabia que hoje existem medicamentos para melhorar a sua saúde e o que mais tinha de informação era o nível de preconceito, para com as pessoas gay que contraem o HIV" (H13).

"[...] eu percebia de uma realidade distante, como algo que jamais fosse chegar em mim, que eu não teria, que jamais você espera ser contaminado, que vai acontecer com você e o estereótipo também, né? [...] que a gente fazia um pré-julgamento daquelas pessoas magérrimas que tem assim um... como eu diria? Que já não tem quase massa muscular, como ficou o estado do Cazuza no filme, né?" (H16).

"Como todo mundo hoje que não tem, que é ignorante e que não sabe de nada e não conhece, que acha que aquela doença vai matar" (H17).

"[...] uma coisa sem resolução e que, sei lá, não dava mais para viver, né? Um negócio desses ia atrapalhar muitas coisas, era isso" (H18).

Sabe-se que o diagnóstico positivo do HIV é algo ameaçador que desencadeia uma série de atitudes e sentimentos no paciente que está infectado pelo vírus, resultando em mudanças na sua vida pessoal, permeando sobre o mesmo o medo, a angústia, gerando uma situação de estresse levando-o a entender a soropositividade como uma sentença de morte (ARAÚJO LFD, et al., 2019).

Entretanto, o modo como os sujeitos compreendem e assimilam a situação que está inserido, conduz seu comportamento individual para o enfrentamento das problemáticas da doença. Diante da escassez de informações sobre o HIV, existe implicação direta no conhecimento e na percepção do risco, contribuindo significativamente para aumentar a vulnerabilidade de contrair o HIV.

A interação simbólica é o processo interpretativo no qual os indivíduos sofrem alterações por meio das relações sociais, sendo que, os mesmos são capazes de pensar e manipular suas acepções em relação aos empecilhos da vida, podendo redefinir seus próprios caminhos (LENZI L, et al., 2018).

O entendimento das causas do HIV e suas formas de transmissão não se traduzem na redução do elevado número de casos na população masculina. Percebeu-se nas falas dos entrevistados a necessidade de novas abordagens e estratégias de prevenção para a infecção do HIV. 
Após o diagnóstico, os homens começaram a ressignificar a doença, o que antes era visto como uma "doença de morte" e sem perspectiva de vida, tornou-se descomplicada e com melhor expectativa de futuro. De acordo com os discursos abaixo:

"Mudou, porque na verdade parece muito mais simples hoje. Porque assim, por exemplo, no meu caso eu tomo só um comprimido só no dia, isso não muda minha rotina, não muda meu estado físico, não muda nada" (H1).

"Mudou! Eu vi que não é tudo aquilo que eu, que a gente tem na cabeça, o preconceito que a gente tem. Eu acho que, é.... só quem tá com HIV que sabe que não é aquilo" (H3).

"Totalmente diferente, hoje eu acredito que existe sim pessoas que tem HIV e são felizes, que podem ser felizes" (H5).

"Mudou bastante, porque quando eu não era soropositivo, eu tinha aquela visão preconceituosa como qualquer uma outra pessoa e que a gente sempre tem aquela visão de que as coisas só acontecem com os outros e nunca acontece com você, mas quando bate na sua porta é diferente, então você começa a ver as coisas de um outro ponto de vista. Então você se torna mais humano, né? Tem muitas coisas que vem pra te ensinar algumas coisas, nem todo o mal vem para te destruir, às vezes ele vem para te ensinar a ser uma pessoa melhor" $(\mathrm{H} 11)$.

"É diferente, muda... É... muda em relação a nós mesmos, como que a gente deve olhar pra gente mesmo, entendeu? Algo que eu não fiz a um tempo atrás, então esse olhar pra mim mesmo muda bastante" (H13).

Os discursos demonstram a capacidade de mudança do significado, que antes era pautado em uma visão estigmatizante do HIV. Assim como a premissa, os participantes dessa pesquisa selecionaram, checaram, suspenderam, reagruparam e transformaram o significado anterior para eles a partir da situação que se encontravam (OLIVEIRA VSD, 2018).

As construções culturais que colocam expectativas sobre o comportamento masculino, torna difícil sua aderência a ações voltadas para a prevenção de doenças, levando dessa forma a descoberta tardia de doenças e falta de adesão a tratamentos. Porém, não se trata apenas da masculinidade hegemônica, o descompromisso do homem com a própria saúde tem base multifatorial e complexa, dado a carência de políticas de saúde que promovam igualdade entre os gêneros (MORA C, et al., 2018).

A mudança de visão, para muitos deve-se à possibilidade de controle da doença, através das medicações e do acompanhamento realizado pelos profissionais de saúde. Existe um aperfeiçoamento no tratamento do HIV/AIDS com o avanço das pesquisas, isto trouxe uma evolução para a sobrevida e a qualidade de vida daqueles que convivem com a doença (LENZI L, et al., 2018).

Entretanto, apesar de existir o avanço no significado do HIV para os homens da pesquisa, ainda permanecem barreiras que os impedem de contar para familiares e sociedade, sendo a mudança ocorrida especificamente para a percepção da longevidade, necessidade do autocuidado, descaracterização do sujeito com HIV.

\section{CONCLUSÃO}

A pesquisa mostrou que existem dificuldades relacionadas ao nível de conhecimento da doença, o estigma e a omissão da soropositividade. Porém, é possível, assim como foi relatado pelos participantes a mudança do significado do HIV, mas para isto é necessário valorizar e estimular as políticas públicas que são realizadas nesse sentido. Conclui-se ainda que, de certa forma, existe relutância no processo de reintegração social devido ao estigma e discriminação relacionada a aceitação da doença. Seria, portanto, imprescindível que fosse incluído programas de saúde voltados para o repasse de informações que interagem com a população propiciando assim, um novo olhar para essas pessoas que convivem com a infecção pelo HIV. 


\section{REFERÊNCIAS}

1. ARAÚJO LFD, et al. Análise da resiliência entre pessoas que vivem com HIV/AIDS: um estudo psicossocial. Psicologia: Teoria e Pesquisa, 2019; 35.

2. BRASIL. Ministério da Saúde: Boletim epidemiológico HIV/ AIDS. Brasília: Ministério da Saúde, 2017. Disponível em: http://www.aids.gov.br/pt-br/pub/2017/boletim-epidemiologico-hivaids-2017

3. BRASIL. Ministério da Saúde. Política Nacional de Atenção Integral a Saúde do Homem. Brasília: Ministério da Saúde, 2009. Disponível em: https://bvsms.saude.gov.br/bvs/publicacoes/politica_nacional_atencao_saude_homem.pdf

4. CAMPOS AER, NERY JSNN. Sentimentos vivenciados pela equipe de enfermagem durante o processo de cuidar de pacientes portadores do HIV/AIDS. Rev. Eletrôn. Atualiza Saúde, 2015; 2(2).

5. CALAZANS GJ. Políticas públicas de saúde e reconhecimento: um estudo sobre prevenção da infecção pelo HIV para homens que fazem sexo com homens. Faculdade de Medicina da Universidade de São Paulo, 2018.

6. CATUNDA C, et al. Qualidade de vida de pessoas vivendo com HIV/aids: efeitos da percepção da doença e de estratégias de enfrentamento. Psicologia: Teoria e Pesquisa, 2016; 32(spe): e32ne218.

7. CEARÁ. Secretaria de Saúde do Estado do Ceará. Boletim epidemiológico. HIV/AIDS. 2021. Disponível em: saude.ce.gov.br/wp-content/uploads/sites/9/2018/06/boletim_epidemiologico_hiv_20212801.pdf

8. COSTA MAR, et al. Qualidade de vida sob a ótica de portadores de HIV/AIDS: perspectivas futuras nas práticas educativas. Rev. Pesqui, 2019; 11(5): 1326-1332.

9. DICARLO AL, et al. 'Men usually say that hiv testing is for women': gender dynamics \& perceptions of hiv testing in Lesotho. Cult Health Sex. 2014; 8(16): 867-882.

10. FARIAS OO, et al. Análise das necessidades de ajuda de homens com HIV que fazem sexo com homens. Revista da Escola de Enfermagem da USP, 2020; 54.

11. FREITAS MIDF, et al. Interações sociais e a adesão à terapia antirretroviral de pessoas vivendo com HIV/AIDS. Rev. Reme, 2017; 1 -5.

12. FRANÇA LCM, et al. As representações sociais da espiritualidade entre homens e mulheres atendidos em um ambulatório de HIV/AIDS. Revista Fragmentos de Cultura-Revista Interdisciplinar de Ciências Humanas, 2020; 29(4): 648-659.

13. GARCIA R, et al. Os sinais traumáticos presentes nos comportamentos sexuais de risco de homens que fazem sexo com homens vivendo com HIV. Boletim-Academia Paulista de Psicologia, 2018; 38.

14. GOMES RRFM, et al. Fatores associados ao baixo conhecimento sobre HIV / AIDS entre homens que fazem sexo com homens no Brasil. Cad. Saúde Pública, 2017; 33(10): e00125515.

15. GUIMARÃES DA, et at. Formação em saúde e extensão universitária: discutindo sexualidade e prevenção de IST/aids. Revista Brasileira de Pesquisa em Saúde, 2017; 19(2): 124-132.

16. JESUS GJ, et al. Dificuldades do viver com HIV/Aids: Entraves na qualidade de vida. Acta paul. Enferm, 2017; 30(3): 301.

17. KNAUTH DR, et al. O diagnóstico do HIV/aids em homens heterossexuais: a surpresa permanece mesmo após mais de 30 anos de epidemia. Cad. Saúde Pública, 2020; 33(10): e00170118.

18. LENZI L, et al. Suporte social e HIV: relações entre características clínicas, sociodemográficas e adesão ao tratamento. Psicologia: Teoria e Pesquisa, 2018; 34.

19. MORA C, et al. HIV/AIDS: sexualidades, subjetividades e políticas. Sexualidad, Salud y Sociedad, 2018; 30: 141-152.

20. NETO JML, et al. Riscos, vulnerabilidades e HIV/Aids entre homens que fazem sexo com homens: uma análise verbal. PUC USP, 2019.

21. OLIVEIRA VSD, et al. Escolhendo descuidar da própria vida apesar de ter consciência da exposição ao HIV/AIDS. Portal Regional da BVS, 2018.

22. REDOSCHI BRL, et al. Uso rotineiro do teste anti-HIV entre homens que fazem sexo com homens: do risco à prevenção. Cad. Saúde Pública, 2017; 33(10): e00014716.

23. SILVA KC. A dor invisível: desvelando experiências de sofrimento emocional de homens heterossexuais que vivem com HIV/Aids. 2018.

24. SILVA LCL, et al. Conhecimento de homens jovens sobre infecção pelo HIV e fatores associados. Revista Baiana de Enfermagem, 2020; 34.

25. SOARES MN, et al. Fatores que influenciam a qualidade de vida de portadores do vírus HIV: uma revisão de literatura. Brazilian Journal of Health Review, 2019; 2(6): 5208-5216.

26. SOUSA LRM, et al. Representações sociais do HIV/Aids por idosos e a interface com a prevenção. Revista Brasileira de Enfermagem, 2019; 72(5): 1129-1136. 